\title{
Postinflammatory Hyperpigmentation Successfully Treated with 1,064-nm Picosecond-Domain Neodymium:Yttrium-Aluminum- Garnet Laser
}

\author{
Hae-Jin Lee ${ }^{1,2}$ \\ Seung Hun Lee ${ }^{1}$ \\ So Young Yoon ${ }^{1}$ \\ Jong Won Lee ${ }^{1}$ \\ Young Koo Kim ${ }^{3}$ \\ Eung $\mathrm{Ho} \mathrm{Choi}^{2}$
}

\footnotetext{
${ }^{1}$ Yonsei A\&B Clinic of Dermatology, Seoul, Korea ${ }^{2}$ Department of Dermatology, Yonsei University Wonju College of Medicine, Seoul, Korea

${ }^{3}$ Yonsei Star Skin \& Laser Clinic, Seoul, Korea
}

Received June 24, 2018

Accepted June 25, 2018

\footnotetext{
Correspondence

Hae-Jin Lee

Department of Dermatology, Yonsei University Wonju College of Medicine, 20 Ilsan-ro, Wonju 26426, Korea

Tel.: +82-33-741-1384

Fax: +82-33-748-2650

E-mail: understndahanmail.net

(c) Korean Society for Laser Medicine and Surgery

(c) This is an open access article distributed under the terms of the Creative Commons Attribution NonCommercial License (http://creativecommons.org/ licenses/by-nc/4.0) which permits unrestricted noncommercial use, distribution, and reproduction in any medium, provided the original work is properly cited.
}

Postinflammatory hyperpigmentation (PIH) is a reactive hypermelanosis of the skin occurring as a result of variable inflammatory processes, such as trauma, and inflammatory dermatosis. Although topical agents are the first-line treatment of $\mathrm{PIH}$, treatment of PIH remains challenging. In this report, we treated a patient with $\mathrm{PIH}$ using picosecond-domain $\mathrm{Nd}$ :YAG lasers. After five sessions of 1,064-nm picosecond-domain $\mathrm{Nd}: Y A G$ laser treatment, the patient presented nearly complete improvement, with no remarkable side effects or recurrence for the duration of 6 months. We suggest that 1,064-nm picosecond-domain $\mathrm{Nd}$ :YAG laser can be effectively and safely used to treat pigmented lesions in the dermis, particularly PIH, in Asian patients.

\section{Key words}

Post-inflammatory hyperpigmentation; Picosecond; Nanosecond; Neodymium:yttrium-aluminum-garnet laser 


\section{INTRODUCTION}

Postinflammatory hyperpigmentation $(\mathrm{PIH})$ is a reactive hypermelanosis of the skin. ${ }^{1} \mathrm{PIH}$ appears as symptomfree macules or patches with variable size and distribution depending on the causative pathology. It usually occurs as a result of an inflammatory dermatosis, such as acne, eczema, drug reactions, burns, chemical peelings, and laser applications. ${ }^{2}$ In $\mathrm{PIH}$, there is either excess melanin production or an abnormal distribution of melanin pigment deposited in the epidermis and/or dermis. ${ }^{1,2}$

There are several modalities of treatment of $\mathrm{PIH}$ with medications and procedures including topical application of hydroquinone, azelaic acid, kojic acid, retinoids, vitamin C, chemical peels, laser therapy, and sunscreens. ${ }^{3}$ Among various treatment modalities for $\mathrm{PIH}$, nanosecond-domain lasers have been most widely utilized including Q-switched ruby laser, the low-dose Q-switched neodymium-doped yttrium aluminum garnet (Nd:YAG) laser, and the fractional 1,550-nm erbium-doped fiber laser. ${ }^{4.5}$ However, there is no published data of therapeutic efficacy of picosecond-domain lasers in $\mathrm{PIH}$.

Herein, we report a case of PIH using 1,064-nm picosecond-domain Nd:YAG lasers. After weekly five sessions of 1,064-nm nanosecond-domain Nd:YAG laser treatment, the patient presented nearly complete improvement in the PIH lesions with no remarkable side effects or recurrence over the duration of 3 months.

\section{CASE REPORT}

A 53-year-old, Korean woman visited our clinic with irregular shaped abrasion wound on the right perinasal area due to fall down. After one week of daily dressing with topical antibiotics, the wound became dark brownish to erythematous patch which showed the features of $\mathrm{PIH}$ (Fig. 1). The patient presented no remarkable treatment history for facial pigmentation within the last six months. The patient was clinically diagnosed with PIH (Fig. 1A and C) and was scheduled to undergo laser treatment. After obtaining written informed consent, the patient was treated with weekly 5 sessions of 1,064-nm picosecond domain Nd:YAG laser treatment (PICOPLUS; Lutronic Corp., Goyang, Koreal was performed at the treatment settings of a $8 \mathrm{~mm}$ spot size, a $0.6 \mathrm{~J} / \mathrm{cm}^{2}$ fluence in a single pulse mode, and a total of approximately 200 shots on the skin lesion. The treated areas were cooled with icepacks, and no prophylactic systemic or topical corticosteroids were prescribed. The patient was recommended to apply sunscreen. At 6 weeks after the first treatment of 1,064-nm picosecond-domain $\mathrm{Nd}$ :YAG laser treatment, the patient presented nearly complete improvement of the PIH lesions without worsening or recurrence (Fig. 1B and D).

\section{DISCUSSION}

Pigmentation abnormalities developing after trauma or cosmetic procedures are always a concern, especially in
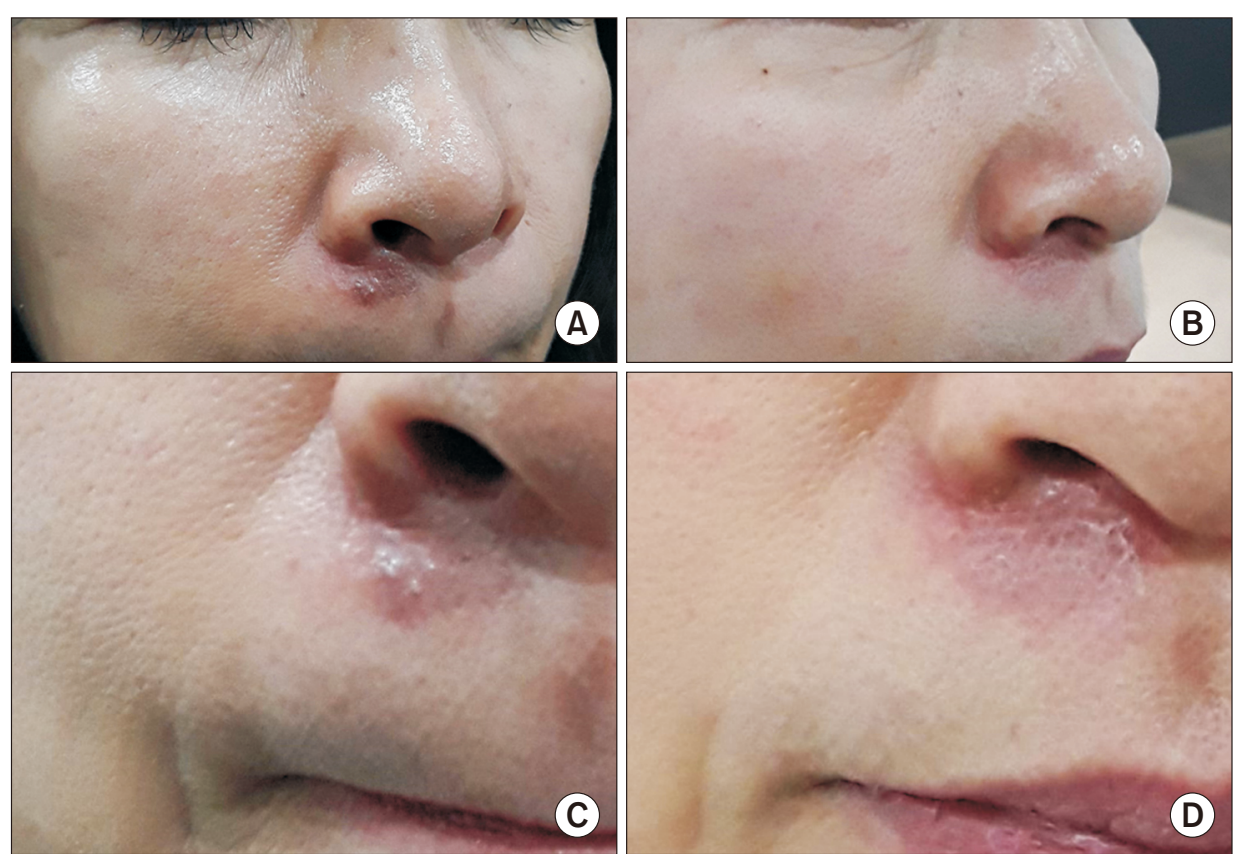
Asians. ${ }^{5}$ Although some cases of PIH tend to be spontaneously regressed, certain forms of PIH need to be treated with several therapeutic attempts, including bleaching creams, several kinds of Q-switched lasers, erbiumdoped fractional photothermolysis system, with various treatment outcomes. There are few reports regarding the efficacy of different laser systems in the treatment of $\mathrm{PIH}$. Oram and Akkaya ${ }^{2}$ have reported refractory PIH treated with fractional CO2 Laser. Cho et al. ${ }^{5}$ have reported successful results with the use of low-energy $\mathrm{Q}$-switched $\mathrm{Nd}$ :YAG laser with nanosecond-domain in three cases of PIH that developed after different laser applications. A 1064-nm Q-switched Nd:YAG with low fluence therapy is easily applicable and the therapeutic trial was revealed to have minimal downtime without post-therapy bleeding or crust formation, and the post-therapy erythema spontaneously resolved within a few hours.

$\mathrm{PIH}$ shares pathologic similarity with tattoo pigmentations. PIH shows hyperpigmentation due to hypermelanosis composed with melanin pigments. In recent studies, picosecond-domain lasers are more efficacious in removing pigment in the form of tattoos with lower risk of side effects than nanosecond-domain lasers. ${ }^{6}$ Smaller size of particles among target chromophores after consecutive treatment would be better responsive to subsequent picosecond-domain laser treatment than nanosecond domain laser treatment. ${ }^{7}$ In this report, we demonstrated near-complete clinical improvement in pigmented lesions after 5 times of treatments with 1,064 nm picoseconddomain Nd:YAG lasers in a Korean patient with PIH. The laser pulses at the picosecond-pulse duration were seemed to effectively destroy target chromophores in our patient. Although further studies are needed to clarify the therapeutic effects of 1,064 $\mathrm{nm}$ picosecond-domain $\mathrm{Nd}$ :YAG lasers, we showed that the picosecond-domain laser treatments might be an effective therapeutic option of the treatment on $\mathrm{PIH}$.

In conclusion, the authors suggest that 1,064-nm picosecond-domain Nd:YAG lasers can be effectively and safely used for treating pigmented lesions in the dermis and epidermis, particularly PIH, in Asian patients. Further prospective, controlled investigations are needed to be followed to compare the efficacy and safety of nanosecond- and picosecond-domain lasers and to optimize the treatment settings for $\mathrm{PIH}$.

\section{REFERENCES}

1. Callender VD, St Surin-Lord S, Davis EC, Maclin M. Postinflammatory hyperpigmentation: etiologic and therapeutic considerations. Am J Clin Dermatol 2011;12:87-99.

2. Oram Y, Akkaya AD. Refractory postinflammatory hyperpigmentation treated fractional CO2 laser. J Clin Aesthet Dermatol 2014;7:42-4.

3. Katz TM, Goldberg LH, Firoz BF, Friedman PM. Fractional photothermolysis for the treatment of postinflammatory hyperpigmentation. Dermatol Surg 2009;35:1844-8.

4. Rokhsar CK, Ciocon DH. Fractional photothermolysis for the treatment of postinflammatory hyperpigmentation after carbon dioxide laser resurfacing. Dermatol Surg 2009;35:535-7.

5. Cho SB, Park SJ, Kim JS, Kim MJ, Bu TS. Treatment of postinflammatory hyperpigmentation using 1064-nm Q-switched $\mathrm{Nd}$ :YAG laser with low fluence: report of three cases. J Eur Acad Dermatol Venereol 2009;23:1206-7.

6. Ross EV. The picosecond revolution and laser tattoo treatments: are shorter pulses really better? Br J Dermatol 2017;176:299-300.

7. Ahn KJ, Zheng Z, Kwon TR, Kim BJ, Lee HS, Cho SB. Pattern analysis of laser-tattoo interactions for picosecond- and nanosecond-domain 1,064-nm neodymium-doped yttriumaluminum-garnet lasers in tissue-mimicking phantom. Sci Rep 2017;7:1533. 